\title{
Uniqueness of Nash equilibrium in private provision of public goods
}

\section{An improved proof}

\author{
Ted C. Bergstrom, Larry Blume and Hal Varian
}

University of Michigan, Ann Arbor, MI 48109-1220, USA

Received January 1992, final version received April 1991

Our 1986 paper 'Private Provision of Public Goods' presents a theorem on the uniqueness of Nash equilibrium in the private provision of public goods. Richard Hirth of the University of Pennsylvania and Clive Fraser of Warwick have independently suggested that although our theorem is correct, our proof is incorrect. Each writer proposes an alternative method of proof for the theorem. With characteristic charity to our own deficiencies, we prefer to view our earlier proof as unduly opaque rather than wrong. Whichever view one takes, a more transparent proof is called for. The proof presented here follows the same outline as our 1986 proof, but explains a step of the argument that was far from obvious in the original proof. This proof uses the notation of the 1986 paper, and refers to 'Facts' that are proved there.

Theorem 3. There is a unique Nash equilibrium, with a uniquely determined quantity of public good and set of contributing consumers.

Suppose that there are two Nash equilibria, with public goods quantities $G$ and $G^{\prime}$, and corresponding sets of contributors $C$ and $C^{\prime}$. Without loss of generality, let $G^{\prime} \leqq G$. Since $G^{\prime}$ is a Nash equilibrium, according to Fact 1 , it must be that $G^{\prime} \geqq f_{i}\left(w_{i}+G^{\prime}{ }_{i}\right)$ for all consumers $i$ (whether or not they belong to $C^{\prime}$ ). In particular, this inequality applies to every consumer in $C$. Therefore $w_{i}+G^{\prime}{ }_{\sim i} \leqq \phi_{i}\left(G^{\prime}\right)$ for all $i \in C$. Summing these inequalities and rearranging terms we have Arbor, MI 48109-1220, USA. 


$$
\sum_{i \in C^{\prime}} w_{i} \leqq \sum_{i \in \mathcal{C}^{\prime}} \phi_{i}\left(G^{\prime}\right)-\sum_{i \in C^{\prime}}\left(G^{\prime}-g_{i}^{\prime}\right)=\sum_{i \in \mathcal{C}} \phi_{i}\left(G^{\prime}\right)-c G^{\prime}+\sum_{i \in \mathcal{C}^{\prime}} g_{i}^{\prime}
$$

But $\sum_{i \in C} g_{i}^{\prime} \leqq \sum_{i \in C^{\prime}} g_{i}^{\prime}=G^{\prime}$. Therefore

$$
\sum_{i \in C} w_{i} \leqq \sum_{i \in C} \phi_{i}\left(G^{\prime}\right)-c G^{\prime}+G^{\prime}=F\left(G^{\prime}, C\right) .
$$

According to Fact 2, $\sum_{i \in C} w_{i}=F(G, C)$. Therefore $F(G, C) \leqq F\left(G^{\prime}, C\right)$. Since $F$ is monotone increasing in $G$, it follows that $G^{\prime} \geqq G$. Therefore $G^{\prime}=G$. The set of contributors is uniquely determined by $G$, so that it must also be that $C=C^{\prime}$. 